Ciência Florestal, Santa Maria, v. 21, n. 2, p. 251-260, abr.-jun., 2011

ISSN 0103-9954

\title{
CARACTERIZAÇÃO DA VEGETAÇÃO E ESPÉCIES PARA RECUPERAÇÃO DE MATA CILIAR, IJUÍ, RS
}

\author{
CHARACTERIZATION OF THE VEGETATION AND SPECIES FOR RECOVERY OF \\ RIPARIAN FOREST, IJUÍ, RS
}

\author{
Angela Luciana de Avila ${ }^{1}$ Maristela Machado Araujo ${ }^{2}$ Solon Jonas Longhi ${ }^{3}$ Ezequiel Gasparin ${ }^{4}$
}

\section{RESUMO}

As matas ciliares constituem corredores ecológicos e possibilitam a preservação da biodiversidade e dos recursos hídricos. A manutenção dessas funções ecológicas, muitas vezes, necessita da recuperação ambiental desses ecossistemas. Dessa forma, este estudo teve como objetivo caracterizar a vegetação arbórea e identificar espécies para a recuperação em áreas de mata ciliar. $\mathrm{O}$ estudo foi realizado em dois fragmentos nas margens direita e esquerda do rio Ijuí, município de Ijuí, RS. A vegetação foi inventariada em dez faixas sistemáticas, com comprimento variando de 17 a $35 \mathrm{~m}$, perpendiculares ao rio, com distância de $50 \mathrm{~m}$ entre $\mathrm{si}$, as quais foram subdivididas em parcelas de $10 \times 10 \mathrm{~m}$, totalizando 21 , onde foram identificados e medidos todos os indivíduos com circunferência a $1,3 \mathrm{~m}$ do solo (CAP) $\geq 15 \mathrm{~cm}$. Os dados obtidos no inventário foram utilizados na caracterização florística e estrutural da vegetação, na análise de agrupamento (TWINSPAN) e serviram de base na indicação de espécies para recuperação. Nos dois fragmentos, foram identificadas 38 espécies distribuídas em vinte famílias botânicas. Os três agrupamentos formados caracterizaram diferentes estágios sucessionais. Para a recuperação ambiental de áreas perturbadas, com características ambientais semelhantes, sugere-se o uso de Ateleia glazioviana, Helietta apiculata, Dalbergia frutescens e Parapiptadenia rigida como facilitadoras da sucessão. O plantio de Eugenia uniflora e Prunus myrtifolia pode auxiliar na atração da fauna dispersora. As espécies Cupania vernalis e Pilocarpus pennatifolius podem ser utilizadas no enriquecimento de áreas em estágio mais avançado da sucessão florestal.

Palavras-chave: Floresta Estacional Decidual; fitossociologia; TWINSPAN; espécies indicadoras.

\begin{abstract}
Riparian forests form ecological corridors and enable the preservation of biodiversity and water resources. The maintenance of these ecological functions often requires the environmental restoration of these ecosystems. Thus, we aimed to characterize the arboreal vegetation and identify species for recovery in areas of riparian forests. The study was conducted in two fragments on the left and right banks of the river Ijuí, in the municipality of Ijuí, RS. The vegetation was inventoried in 10 systematic strips, of lengths from 15-35 m, perpendicular to the river, with a distance of $50 \mathrm{~m}$, which were subdivided into plots of 10 x $10 \mathrm{~m}$, totaling 21 , in which were identified and measured all individuals with girth breast height (GBH) $\geq 15 \mathrm{~cm}$. Data from the inventory were used in the floristic and structural characterization of vegetation, in a clustering analysis (TWINSPAN) and supplied a basis for the indication of species for recovery. We identified 38 species distributed in 20 families. The three groups formed showed different succession stages.
\end{abstract}

1. Engenheira Florestal, Mestre em Engenharia Florestal, Professora do Curso de Engenharia Florestal da Universidade do Oeste de Santa Catarina (UNOESC), Campus de Xanxerê, Rua Dirceu Giordani, 696, Bairro Jardim Universitário, CEP 89820-000, Xanxerê (SC). angeladeavila@gmail.com

2. Engenheira Florestal, Dr ${ }^{\mathrm{a}}$, Professora Adjunta do Departamento de Ciências Florestais, Centro de Ciências Rurais, Universidade Federal de Santa Maria, Av. Roraima, 1000, CEP 97105-900, Santa Maria (RS). araujo.maristela@gmail.com

3. Engenheiro Florestal, Dr., Professor Titular do Departamento de Ciências Florestais, Centro de Ciências Rurais, Universidade Federal de Santa Maria, Av. Roraima, 1000, CEP 97105-900, Santa Maria (RS). longhi.solon@gmail.com

4. Engenheiro Florestal, Mestrando do Programa de Pós-graduação em Engenharia Florestal, Centro de Ciências Rurais, Universidade Federal de Santa Maria, Av. Roraima, 1000, CEP 97105-900, Santa Maria (RS). Bolsista da Capes. ezequiel_gasparin@hotmail.com

Recebido para publicação em 26/08/2009 e aceito em 5/08/2010 
For the environmental recovery of altered areas, with similar environmental characteristics, it is suggested the use of Ateleia glazioviana, Helietta apiculata, Dalbergia frutescens and Parapiptadenia rigida as these facilitate succession. The planting of Eugenia uniflora and Prunus myrtifolia can help in attracting disperser fauna. Cupania vernalis and Pilocarpus pennatifolius may be used for the enrichment of areas in a more advanced stage of forest succession.

Keywords: Seasonal Deciduous Forest; phytossociology; TWINSPAN; indicator species.

\section{INTRODUÇÃO}

Nas últimas décadas, o impacto da ocupação humana e a destruição da Mata Atlântica aumentaram, gerando elevada fragmentação nesse bioma. A área original dos habitats foi reduzida, ocasionando mudanças nos padrões de composição e abundância de espécies e, consequentemente, nos processos ecológicos das comunidades. Assim, identifica-se a necessidade de promover a recuperação de áreas para interligar os fragmentos (RAMBALDI e OLIVEIRA, 2005; SANTOS, 2007), superando o isolamento e os efeitos prejudiciais da fragmentação.

Nesse sentido, as matas ciliares constituem corredores ecológicos, pois propiciam o deslocamento e a continuidade do fluxo gênico da fauna e flora, proporcionando a manutenção de espécies e a variabilidade entre populações (MARIOT, 2007). Além disso, proporcionam a preservação dos mananciais hídricosfluviais, os quais desempenham importante função social e ecológica por meio do abastecimento público, irrigação, uso industrial, preservação da biodiversidade e manutenção do equilíbrio ecológico.

Entretanto, no Rio Grande do Sul (RS), os processos de ocupação territorial e desenvolvimento econômico, muitas vezes, acarretaram gradativa ou integral supressão da vegetação ao longo de rios e arroios (RIO GRANDE DO SUL, 2007). Dessa forma, em razão da relevância das matas ciliares, alterações sobre elas constituem um desafio para a recuperação ambiental.

A aplicação de métodos de recuperação pressupõe o conhecimento detalhado do ecossistema, sendo os estudos fitossociológicos importantes para caracterizar a vegetação, pois permitem analisar a sua estrutura e identificar espécies potenciais para recuperação de áreas em cada região (FERRETTI, 2002; ATTANASIO, 2008).

A descrição florística fornece um indicativo da composição de espécies no ecossistema, bem como, permite identificar a área mínima a ser amostrada. A caracterização por meio de parâmetros que representam a estrutura horizontal como densidade, frequência, dominância e índice de valor de importância (IVI) permite identificar a estrutura da vegetação e a hierarquização das espécies (FELFILI e VENTUROLI, 2000).

Além disso, cada espécie apresenta um intervalo de tolerância para os fatores ambientais cujos indivíduos, geralmente, vivem em habitat adequado ao seu desenvolvimento, existindo uma interação entre a distribuição da vegetação e as condições ambientais (BOURGERON, 1983; RICKLEFS e MILLER, 1999). Assim, a vegetação pode apresentar variações formando mosaicos em sua distribuição, alterando sua composição e estrutura de acordo com as características de cada ambiente.

As técnicas de classificação buscam agrupar amostras que apresentam características em comum, determinando a formação de agrupamentos com base na composição florística (MATTEUCCI e COLMA, 1982). A identificação de grupos ou padrões diferenciados na vegetação pode refletir padrões sucessionais, associação entre espécies e as respectivas espécies características, fornecendo informações importantes para orientar o planejamento de técnicas de recuperação ambiental.

O método TWINSPAN (Two-way Indicator Species Analysis) considera que cada agrupamento é diferenciado do outro por meio das espécies que os caracterizam, tendo como base a presença e ausência de pseudoespécies na parcela (KENT e COKER, 1992). Por sua vez, a pseudoespécie representa as diferentes amplitudes de densidade da espécie analisada. Assim, a classificação da vegetação pelo TWINSPAN permite identificar agrupamentos e suas respectivas espécies indicadoras, as quais apresentam ocorrência restrita ao grupo.

As informações sobre as florestas ripárias, no estado do Rio Grande do Sul, são escassas e dispersas, podendo citar os estudos realizados por Durlo et al. (1982), Kilka (2002), Araujo et al. (2004) e Budke et al. (2004). Dessa forma, visando contribuir com informações sobre as matas ciliares do Estado, este estudo teve como objetivos: a)

Ci. Fl., v. 21, n. 2, abr.-jun., 2011 
caracterizar a vegetação arbórea e identificar a presença de agrupamentos que possam auxiliar na seleção de espécies e; b) indicar espécies para a recuperação de áreas perturbadas de mata ciliar.

\section{MATERIAL E MÉTODO}

O estudo foi desenvolvido em dois fragmentos de mata ciliar, nas margens direita e esquerda do rio Ijuí, município de Ijuí, RS. A área localiza-se nas coordenadas $28^{\circ} 17,46^{\prime} \mathrm{S}$ e $53^{\circ} 52,59^{\prime} \mathrm{W}$ e a altitude se situa em torno de $290 \mathrm{~m}$. Os fragmentos apresentam formato retangular e a largura máxima encontrada foi, aproximadamente, 35 m. A configuração dos fragmentos, juntamente com sua adjacência à área agrícola, permite inferir que a dinâmica da vegetação pode ser influenciada pelo efeito de borda que possibilita maior entrada de luz e, consequentemente, diminuição no teor de umidade e aumento da temperatura no interior da vegetação.

Os solos predominantes da região são do tipo latossolo vermelho distrófico típico, com acidez acentuada e baixa saturação por bases $(<50 \%)$ (STRECK et al., 2008). Conforme a classificação de Köppen, a região apresenta clima do tipo 'Cfa' (Subtropical úmido), caracterizado por apresentar temperatura média do mês mais frio entre -3 e $18^{\circ} \mathrm{C}$ e, do mês mais quente, temperatura média superior a $22^{\circ} \mathrm{C}$, com chuvas distribuídas durante o ano todo (MORENO, 1961).

A formação vegetal característica da região é a Floresta Estacional Decidual, na qual é possível distinguir quatro estratos: árvores superiores e emergentes, árvores com alturas semelhantes, arvoretas e arbustos. As espécies que conferem a característica de caducifolia são: Apuleia leiocarpa (grápia), Cordia trichotoma (louro), Balfourodendron riedelianum (pau-marfim) e Enterolobium contortisiliquum (timbaúva). Das espécies perenifólias pode-se destacar: Nectandra megapotamica (canela-preta), Ocotea puberula (canela-guaicá) e Cordia americana (guajuvira) (QUADROS e PILLAR, 2002; LEITE, 2002).

A amostragem da vegetação foi realizada por meio de faixas sistemáticas e perpendiculares à margem do rio. A distância entre as faixas foi de $50 \mathrm{~m}$ e a largura de $10 \mathrm{~m}$, sendo que cada faixa foi subdividida em parcelas $10 \times 10 \mathrm{~m}$. O total de parcelas amostradas foi de 21 , dez locadas no fragmento 1 e 11 no fragmento 2 .

$\mathrm{O}$ inventário da vegetação utilizou como critério de inclusão a circunferência a 1,3 m do solo (CAP) igual ou maior que $15 \mathrm{~cm}$, mesurando CAP e altura total. A verificação da suficiência amostral da vegetação foi realizada pela análise gráfica da curva do número acumulado de espécies $\mathrm{x}$ área amostrada (parcela) (curva espécie-área). Segundo Brower e Zar (1984), o número de amostras é considerado suficiente quando a curva tende a estabilização. A identificação das espécies foi realizada por meio de coleta de material botânico e consulta ao Herbário do Departamento de Ciências Florestais (HDCF) da UFSM. A nomenclatura das espécies segue a proposta do Angiosperm Philogeneny Group (APG) (APG II, 2002 apud SOBRAL et al., 2006).

A caracterização da vegetação foi realizada pela análise da composição florística, estrutura horizontal e índice de diversidade de Shannon $\left(\mathrm{H}^{\prime}\right)$. Além disso, com base nos dados obtidos foi construída uma matriz 21 x 29, constituída de 21 parcelas (linhas) e 29 espécies (colunas), relacionando a densidade para cada espécie dentro de cada parcela e desconsiderando as espécies com menos de três indivíduos amostrados. Essa matriz foi utilizada na classificação dos dados, por meio da análise de agrupamento pelo método TWINSPAN (Two-way Indicator Species Analysis). Os níveis de corte utilizados foram 3, 5, 7, 10 e 15. Segundo Araujo et al. (2004), os pontos de corte são utilizados para caracterizar as pseudoespécies sendo que, por exemplo, a espécie Ateleia glazioviana seria dividida em seis pseudoespécies (At $g$ 1, 2, 3, 4, 5 e 6), assim, a pseudoespécie 3 (At $g$ 3) representa de cinco a seis indivíduos em determinada parcela.

Os resultados foram confrontados com observações a campo, visando a comprovar a presença dos agrupamentos. O método TWINSPAN também fornece a variância explicada pela análise por meio do autovalor da divisão, além das espécies indicadoras e preferenciais de cada grupo. Posteriormente, os agrupamentos formados foram analisados em relação à composição florística e estrutural, buscando definir diferenças biológicas presentes em cada agrupamento. A análise da estrutura horizontal da vegetação foi realizada com base nos seguintes parâmetros: densidade, dominância e frequência, em suas formas absolutas e relativas, e pelo índice de valor de importância (IVI) para cada espécie.

A indicação de espécies para recuperação de áreas perturbadas considerou os resultados obtidos neste estudo e revisão bibliográfica sobre as espécies, considerando suas características ecológicas 
(REITZ et al., 1983; BACKES e IRGANG, 2002; CARVALHO, 2003; RIO GRANDE DO SUL, 2007).

\section{RESULTADOS E DISCUSSÃO}

A análise da curva espécie-área (Figura 1) permite identificar que o número de espécies tende a estabilizar partindo de $1.600 \mathrm{~m}^{2}$ (parcela 16). Assim, pode-se inferir que a amostragem utilizada no estudo foi satisfatória para caracterizar a vegetação nos fragmentos estudados. Rosa et al. (2008) em estudo realizado na Reserva Ecológica Capão de Tupanciretã, Tupanciretã, RS, também observaram tendência à estabilização da curva após $1.800 \mathrm{~m}^{2}$, ou seja, com valor de amostragem semelhante ao encontrado no presente estudo.

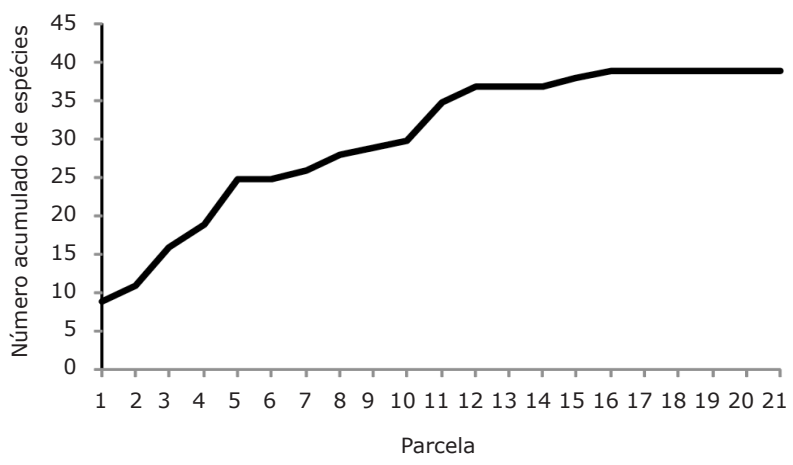

FIGURA 1: Curva espécie-área dos indivíduos amostrados em fragmentos de mata ciliar, Ijuí, RS, Brasil.

FIGURE 1: Species-area curve of the individuals sampled in fragments of riparian forest, Ijuí, RS, Brazil.

Na composição florística, foram observadas 38 espécies distribuídas em vinte famílias botânicas, sendo amostrados 463 indivíduos. As famílias mais representativas foram: Fabaceae, Myrtaceae, Rutaceae e Sapindaceae (Tabela 1). Entre estas, a família Myrtaceae foi observada em outros estudos como a mais representativa na mata ciliar, em áreas de Floresta Estacional Decidual (ARAUJO et al., 2004; SEMA/UFSM-RS, 2002). As famílias Salicaceae e Bignoniaceae foram representadas apenas com uma espécie e um indivíduo. O índice de diversidade de Shannon (H') encontrado para a área foi 3,02. Em levantamento realizado próximo à região deste estudo, Vaccaro e Longhi (1995) identificaram índice de Shannon de 3,71, o qual, segundo os autores indica elevada diversidade para o tipo florestal estudado.

A classificação dos dados da vegetação, pelo TWINSPAN, dividiu a área em três agrupamentos (Figura 2), com autovalores de 0,6476 para a primeira divisão que deu origem ao Agrupamento 1 e de 0,5146 para a segunda divisão, gerando os Agrupamentos 2 e 3. Segundo Felfili e Venturoli (2000) autovalores superiores a 0,3 indicam que as divisões têm significado ecológico.

O Agrupamento 1 apresentou Ateleia glazioviana (timbó) como espécie indicadora, a qual é descrita como pioneira, agressiva e característica de vegetação secundária (REITZ et al., 1983). Entre as espécies preferenciais do primeiro grupo, pode-se destacar Helietta apiculata, Sebastiania commersoniana e Luehea divaricata, as quais são comumente observadas em mata ciliar (REITZ et al., 1983; BACKES e IRGANG, 2002). Estas, apesar de serem espécies de estágio inicial de sucessão (RIO GRANDE DO SUL, 2007), apresentam potencial para permanecer durante maior período na floresta, quando comparadas com Ateleia glazioviana que, conforme Carvalho (2003), apresenta ciclo de vida curto e dificilmente ocorre no interior da floresta. As parcelas pertencentes a esse agrupamento são adjacentes à área agrícola, e a presença de espécies características de estágios iniciais da sucessão pode estar associada à maior alteração e influência do efeito de borda que, segundo Attanasio (2008), propicia elevada incidência de luz e maiores variações na temperatura.

O Agrupamento 1 mostrou uma densidade total de 2.244 indivíduos por hectare, sendo amostradas 25 espécies distribuídas em 17 famílias botânicas. As famílias mais representativas do grupo foram Fabaceae (IVI $=37,7 \%$ ) e Rutaceae (IVI $=14,7 \%$ ). A maior representatividade da família Fabaceae, nesse agrupamento, constitui informação importante para a recuperação, considerando que a ela se caracteriza por apresentar espécies fixadoras de nitrogênio pela associação simbiótica com bactérias do gênero Rhizobium, o que por sua vez, melhora as condições edáficas para o estabelecimento de espécies mais exigentes. A predominância da Família Fabaceae em comunidades de estágio sucessional menos avançado também foi observada por Vaccaro et al. (1999), em estudo sobre a caracterização de três estágios sucessionais na Floresta Estacional Decidual, Santa Tereza, RS.

Ci. Fl., v. 21, n. 2, abr.-jun., 2011 
TABELA 1: Famílias e espécies amostradas em fragmentos de mata ciliar, Ijuí, RS, Brasil.

TABLE 1: Family and species sampled in fragments of Riparian forest, Ijuí, RS, Brazil.

\begin{tabular}{|c|c|c|}
\hline Família & Nome científico & Nome comum \\
\hline Annonaceae & Rollinia salicifolia Schltdl. & Ariticum \\
\hline Apocynaceae & Aspidosperma australe Müll. Arg. & Pitiá \\
\hline Arecaceae & Syagrus romanzoffiana (Cham.) Glassman & Jerivá \\
\hline Bignoniaceae & Jacaranda micrantha Cham. & Caroba \\
\hline Boraginaceae & Cordia americana (L.) Gottschling \& J.E. Mill. & Guajuvira \\
\hline Ebenaceae & Diospyros inconstans Jacq. & Maria-preta \\
\hline Erythroxylaceae & Erythroxylum deciduum A.St.-Hil. & Cocão \\
\hline Euphorbiaceae & Sebastiania commersoniana (Baill.) L.B.Sm. \& Downs & Branquilho \\
\hline \multirow[t]{8}{*}{ Fabaceae } & Albizia niopoides (Spruce ex Benth.)Burkart & Angico-branco \\
\hline & Ateleia glazioviana Baill. & Timbó \\
\hline & Bauhinia forficata Link & Pata-de-vaca \\
\hline & Dalbergia frutescens (Vell.) Britton & Rabo-de-bugio \\
\hline & Holocalyx balansae Micheli & Alecrim \\
\hline & Machaerium paraguariense Hassl. & Canela-do-brejo \\
\hline & Myrocarpus frondosus Allemão & Cabreúva \\
\hline & Parapiptadenia rigida (Benth.) Brenan & Angico-vermelho \\
\hline \multirow[t]{2}{*}{ Lauraceae } & Nectandra megapotamica (Spreng.) Mez & Canela-preta \\
\hline & Ocotea pulchella (Nees) Mez & Canela-lajeana \\
\hline Loganiaceae & Strychnos brasiliensis (Spreng.) Mart. & Esporão-de-galo \\
\hline Malvaceae & Luehea divaricata Mart. \& Zucc. & Açoita-cavalo \\
\hline \multirow[t]{2}{*}{ Meliaceae } & Trichilia catigua A.Juss. & Catiguá \\
\hline & Trichilia elegans A.Juss. & Pau-de-ervilha \\
\hline \multirow[t]{4}{*}{ Myrtaceae } & Campomanesia guazumifolia (Cambess.) O.Berg & Sete-capotes \\
\hline & Eugenia pyriformis Cambess. & Uvaia \\
\hline & Eugenia uniflora $\mathrm{L}$. & Pitanga \\
\hline & Myrcianthes pungens (O.Berg) D.Legrand & Guabiju \\
\hline Rosaceae & Prunus myrtifolia (L.) Urb. & Pessegueiro-bravo \\
\hline \multirow[t]{4}{*}{ Rutaceae } & Balfourodendron riedelianum (Engl.) Engl. & Guatambú \\
\hline & Helietta apiculata Benth. & Canela-de-veado \\
\hline & Pilocarpus pennatifolius Lem. & Cutia \\
\hline & Zanthoxylum petiolare A.St.-Hil. \& Tul. & Mamica-de-cadela \\
\hline Salicaceae & Casearia decandra Jacq. & Guaçatunga \\
\hline \multirow[t]{3}{*}{ Sapindaceae } & Allophylus edulis (A. St.-Hil., Cambess. \& A. Juss.) Radlk. & Chal-chal \\
\hline & Cupania vernalis Cambess. & Camboatá-vermelho \\
\hline & Diatenopteryx sorbifolia Radlk. & Maria-preta \\
\hline Sapotaceae & Chrysophyllum marginatum (Hook. \& Arn.) Radlk. & Aguaí-leiteiro \\
\hline \multirow[t]{2}{*}{ Solanaceae } & Brunfelsia australis Benth. & Primavera \\
\hline & Solanum sanctaecatharinae Dunal & Joá-manso \\
\hline
\end{tabular}


Avila, A. L. et al.

\begin{tabular}{|c|c|}
\hline \multicolumn{2}{|c|}{ Conjunto de Parcelas } \\
\hline & Autovalor da $1^{\text {a }}$ Divisão: 0,6476 \\
\hline & Agrupamento 1 \\
\hline $\begin{array}{l}\text { Parcelas: } \\
3,4,5,6,7,8,9,10,12,13,14,17\end{array}$ & $\begin{array}{l}\text { Parcelas: } \\
1,2,11,15,16,18,19,20,21\end{array}$ \\
\hline $\begin{array}{l}\text { Espécies Indicadoras: } \\
\text { Não foram detectadas espécies indicadoras }\end{array}$ & $\begin{array}{l}\text { Espécies Indicadoras: } \\
\text { Ateleia glazioviana } 2\end{array}$ \\
\hline 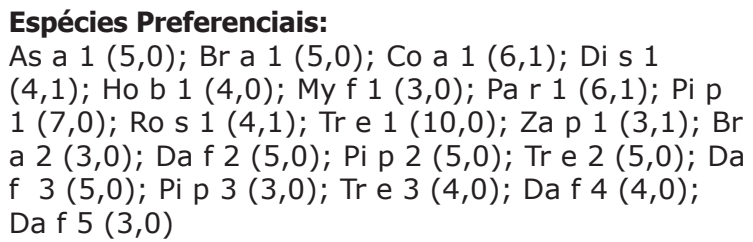 & $\begin{array}{l}\text { Espécies Preferenciais: } \\
\text { Al e } 1(1,3) ; \text { At g } 1(2,8) ; \text { Ja m } 1(0,2) ; \text { Lu d } 1 \\
(0,3) ; \mathrm{Se} \mathrm{C} 1(1,5) ; \mathrm{Sy} 1(3,6) ; \text { At g } 2(0,8) ; \mathrm{He} \text { a } \\
2(2,6) ; \text { Lu d } 2(0,2) ; \mathrm{Se} \mathrm{c} 2(0,3) ; \text { At g } 3(0,7) ; \mathrm{He} \\
\text { a } 3(1,3) ; \text { Se c } 3(0,2) ; \text { At g } 4(0,4) ; \text { He a } 4(0,3) ; \\
\text { At g } 5(0,2)\end{array}$ \\
\hline
\end{tabular}

\begin{tabular}{|c|c|c|c|}
\hline & \multicolumn{2}{|c|}{ Autovalor da 2a Divisão: 0,5146 } & \multirow[b]{2}{*}{ Agrupamento 3} \\
\hline & Agrupamento 2 & & \\
\hline \multicolumn{2}{|l|}{$\begin{array}{l}\text { Parcelas: } \\
5,6,7,8,9,10\end{array}$} & \multicolumn{2}{|l|}{$\begin{array}{l}\text { Parcelas: } \\
3,4,12,13,14,17\end{array}$} \\
\hline \multirow{2}{*}{\multicolumn{2}{|c|}{$\begin{array}{l}\text { Espécies Indicadoras: } \\
\text { Pilocarpus pennatifolius } 1 \\
\text { Holocalyx balansae } 1\end{array}$}} & \multicolumn{2}{|l|}{$\begin{array}{l}\text { Espécies Indicadoras: } \\
\text { Dalbergia frutescens } 1\end{array}$} \\
\hline & & \multirow{2}{*}{\multicolumn{2}{|c|}{ 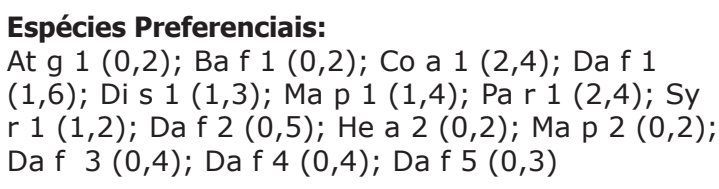 }} \\
\hline \multicolumn{2}{|c|}{$\begin{array}{l}\text { Espécies Preferenciais: } \\
\mathrm{Br} \text { a } 1(4,1) ; \text { Ho b } 1(4,0) ; \text { My p } 1(2,0) ; \text { My f } 1 \\
(2,1) ; \operatorname{Pi~p~} 1(6,1) ; \operatorname{Tr} \text { e } 1(2,0) ; \operatorname{Za~p~} 1(2,1) ; \operatorname{Br} \text { a } 2 \\
(3,0) ; \operatorname{Pi~p~} 2(4,0) ; \operatorname{Tr} \text { e } 2(5,2) ; \operatorname{Pi~p~} 3(3,0) ; \operatorname{Tr} \text { e } 3 \\
(3,1) \text { Pi p } 4(2,0)\end{array}$} & & \\
\hline
\end{tabular}

Abreviaturas: Al n (Albizia niopoides); Al e (Allophylus edulis); As a (Aspidosperma australe); At g (Ateleia glazioviana); $\mathrm{Ba} f$ (Bauhinia forficata); $\mathrm{Br}$ a (Brunfelsia australis); Co a (Cordia americana); Cu v (Cupania vernalis); Da f (Dalbergia frutescens); Di s (Diatenopteryx sorbifolia); Di i (Diospyros inconstans); Eu p (Eugenia pyriformis); He a (Helietta apiculata); Ho b (Holocalyx balansae); Ja m (Jacaranda micrantha); Lu d (Luehea divaricata); Ma $\mathrm{p}$ (Machaerium paraguariense); My $\mathrm{p}$ (Myrcianthes pungens); My f (Myrocarpus frondosus); Ni (Nao identificada); Pa r (Parapiptadenia rigida); Pi p (Pilocarpus pennatifolius); Pr m (Prunus myrtifolia); Ro s (Rollinia salicifolia); Se c (Sebastiania commersoniana); Sy r (Syagrus romanzoffiana); Tr c (Trichilia catigua); Tr e (Trichilia elegans); Za p (Zanthoxylum petiolare).

Observação: O número depois da abreviatura refere-se à pseudoespécie (nível de corte). O número entre parênteses refere-se às parcelas onde estão contidas as pseudoespécies, em ambos os lados da divisão.

FIGURA 2: Classificação das unidades amostrais caracterizando três agrupamentos em fragmentos de mata ciliar, Ijuí, RS, Brasil.

FIGURE 2: Classification of the units of samples characterizing three groupings in fragments of Riparian forest, Ijuí, RS, Brazil.

As espécies melhor hierarquizadas no Agrupamento 1 foram: Ateleia glazioviana, Helietta apiculata, Syagrus romanzoffiana e Sebastiania commersoniana, as quais perfazem $60,9 \%$ do valor de importância (Tabela 2). Entre estas, Ateleia glazioviana corresponde a 30,2\%, indicando sua predominância na fisionomia do agrupamento. Em estudo realizado pelo Inventário Florestal Contínuo do RS, Helietta apiculata foi descrita como espécie abundante nos estágios inicias de sucessão em florestas no domínio da bacia do rio Ijuí (SEMA/UFSM-RS, 2002).

As espécies indicadoras do Agrupamento 2 foram Pilocarpus pennatifolius e Holocalyx balansae, consideradas de sub-bosque e clímax respectivamente (REITZ et al., 1983; VACCARO et al., 1999). Esse agrupamento demonstrou o melhor estado de conservação, possivelmente, em razão da dificuldade de acesso pela maior declividade, o que inibiu a interferência antrópica. Nesse agrupamento, foram encontrados 1.850 indivíduos por hectare, 
distribuídos em 23 espécies e 11 famílias, dentre as quais, as mais representativas da área foram: Fabaceae, Rutaceae e Meliaceae. As espécies com maior valor de importância foram: Pilocarpus pennatifolius (VI $=15,03 \%)$, Trichilia elegans $(12,74 \%)$, Cordia americana (10,05\%), Holocalyx balansae (8,99\%), Parapiptadenia rigida $(8,53 \%)$ e
Brunfelsia australis (7,32\%) (Tabela 2). Identificase, nesse agrupamento, a presença de elementos indicadores de um estágio sucessional mais avançado, como o alecrim (Holocalyx balansae) e a cutia (Pilocarpus pennatifolius), espécies características do interior de florestas primárias (REITZ et al., 1983).

TABELA 2: Estrutura horizontal dos três grupos em fragmentos de mata ciliar, Ijuí, RS, Brasil.

TABLE 2: Horizontal structure of the three groups in fragments of Riparian forest, Ijuí, RS, Brazil.

\begin{tabular}{|c|c|c|c|c|c|c|c|c|c|c|c|c|}
\hline \multirow{2}{*}{ Nome científico } & \multicolumn{4}{|c|}{ Agrupamento 1} & \multicolumn{4}{|c|}{ Agrupamento 2} & \multicolumn{4}{|c|}{ Agrupamento 3} \\
\hline & $\mathrm{DR}^{*}$ & DoR & FR & $\mathrm{IVI} \%$ & DR & DoR & FR & $\mathrm{IVI} \%$ & DR & DoR & FR & $\mathrm{IVI} \%$ \\
\hline Albizia niopoides & & & & & 0,90 & 0,16 & 2,00 & 1,02 & 1,31 & 0,66 & 1,92 & 1,30 \\
\hline Allophylus edulis & 3,47 & 1,09 & 4,84 & 3,13 & 0,90 & 0,34 & 2,00 & 1,08 & & & & \\
\hline Aspidosperma australe & & & & & 1,80 & 3,70 & 4,00 & 3,17 & 5,23 & 11,18 & 5,77 & 7,39 \\
\hline Ateleia glazioviana & 29,7 & 47,96 & 12,90 & 30,19 & & & & & 2,61 & 9,97 & 3,85 & 5,48 \\
\hline $\begin{array}{l}\text { Balfourodendron } \\
\text { riedelianum }\end{array}$ & & & & & 0,90 & 0,19 & 2,00 & 1,03 & & & & \\
\hline Bauhinia forficata & 0,99 & 0,65 & 3,23 & 1,62 & & & & & 1,31 & 0,36 & 3,85 & 1,84 \\
\hline Brunfelsia australis & & & & & 11,71 & 2,25 & 8,00 & 7,32 & 1,31 & 0,61 & 1,92 & 1,28 \\
\hline Campomanesia guazumifolia & & & & & 1,80 & 1,60 & 4,00 & 2,47 & 0,65 & 0,16 & 1,92 & 0,91 \\
\hline Casearia decandra & 0,50 & 0,10 & 1,61 & 0,74 & & & & & & & & \\
\hline Chrysophyllum marginatum & 0,50 & 0,33 & 1,61 & 0,81 & & & & & & & & \\
\hline Cordia americana & 1,49 & 2,22 & 1,61 & 1,77 & 1,80 & 24,34 & 4,00 & 10,05 & 5,88 & 6,85 & 7,69 & 6,81 \\
\hline Cupania vernalis & 1,49 & 0,89 & 4,84 & 2,41 & 3,60 & 0,95 & 6,00 & 3,52 & 2,61 & 1,57 & 3,85 & 2,68 \\
\hline Dalbergia frutescens & 2,48 & 1,10 & 6,45 & 3,34 & 0,90 & 1,12 & 2,00 & 1,34 & 39,87 & 23,30 & 11,54 & 24,90 \\
\hline Diatenopteryx sorbifolia & 0,99 & 0,90 & 1,61 & 1,17 & 0,90 & 1,70 & 2,00 & 1,53 & 3,92 & 8,16 & 5,77 & 5,95 \\
\hline Diospyros inconstans & 3,96 & 1,60 & 1,61 & 2,39 & & & & & & & & \\
\hline Erythroxylum deciduum & 0,50 & 0,20 & 1,61 & 0,77 & & & & & & & & \\
\hline Eugenia pyriformis & 1,49 & 0,55 & 1,61 & 1,22 & & & & & & & & \\
\hline Eugenia uniflora & 0,99 & 0,23 & 1,61 & 0,94 & & & & & & & & \\
\hline Helietta apiculata & 17,8 & 9,07 & 11,29 & 12,73 & 1,80 & 4,63 & 4,00 & 3,48 & 6,54 & 2,90 & 5,77 & 5,07 \\
\hline Holocalyx balansae & & & & & 6,31 & 12,67 & 8,00 & 8,99 & & & & \\
\hline Jacarandá micrantha & 0,99 & 0,52 & 3,23 & 1,58 & & & & & & & & \\
\hline Luehea divaricata & 5,45 & 2,65 & 4,84 & 4,31 & & & & & & & & \\
\hline Machaerium stipitatum & 4,95 & 5,21 & 8,06 & 6,07 & 0,90 & 0,38 & 2,00 & 1,09 & 5,23 & 7,42 & 7,69 & 6,78 \\
\hline Myrcianthes pungens & & & & & 2,70 & 0,59 & 4,00 & 2,43 & & & & \\
\hline Myrocarpus frondosus & & & & & 3,60 & 8,58 & 4,00 & 5,39 & 0,65 & 0,44 & 1,92 & 1,00 \\
\hline Nectandra megapotamica & & & & & & & & & 0,65 & 0,20 & 1,92 & 0,92 \\
\hline Ocotea pulchella & 0,50 & 0,17 & 1,61 & 0,76 & & & & & & & & \\
\hline Parapiptadenia rigida & 0,99 & 0,25 & 1,61 & 0,95 & 1,80 & 19,80 & 4,00 & 8,53 & 3,92 & 10,21 & 7,69 & 7,27 \\
\hline Pilocarpus pennatifolius & & & & & 25,23 & 7,86 & 12,00 & 15,03 & 1,31 & 0,25 & 1,92 & 1,16 \\
\hline Prunus myrtifolia & 2,97 & 2,15 & 1,61 & 2,24 & 0,90 & 0,24 & 2,00 & 1,05 & 0,65 & 0,19 & 1,92 & 0,92 \\
\hline Rollinia salicifolia & 0,50 & 0,27 & 1,61 & 0,79 & & & & & 2,61 & 3,32 & 3,85 & 3,26 \\
\hline Sebastiania commersoniana & 10,4 & 8,42 & 8,06 & 8,96 & & & & & 0,65 & 0,19 & 1,92 & 0,92 \\
\hline Solanum sanctae-catharinae & 0,50 & 0,08 & 1,61 & 0,73 & & & & & & & & \\
\hline Strychnos brasiliensis & & & & & & & & & 1,31 & 0,42 & 1,92 & 1,22 \\
\hline Syagrus romanzoffiana & 4,95 & 12,38 & 9,68 & 9,00 & 0,90 & 1,99 & 2,00 & 1,63 & 3,27 & 8,54 & 3,85 & 5,22 \\
\hline Trichilia catigua & & & & & 3,60 & 1,11 & 4,00 & 2,90 & & & & \\
\hline Trichilia elegans & & & & & 23,42 & 2,79 & 12,00 & 12,74 & 7,19 & 2,67 & 7,69 & 5,85 \\
\hline Zanthoxylum petiolare & 1,49 & 1,04 & 1,61 & 1,38 & 1,80 & 2,73 & 4,00 & 2,84 & 0,65 & 0,12 & 1,92 & 0,90 \\
\hline Total geral & 100,0 & 100,0 & 100,0 & 100,0 & 100,0 & 100,0 & 100,0 & 100,0 & 100,0 & 100,0 & 100,0 & 100,0 \\
\hline
\end{tabular}

Em que: $\mathrm{DR}=$ Densidade relativa; DoR $=$ Dominância relativa; $F R=$ Frequência relativa; IVI\% $=$ Índice de valor de importância. 
Avila, A. L. et al.

O Agrupamento 3 apresentou como espécie indicadora Dalbergia frutescens, considerada pioneira e de estágio inicial (RIO GRANDE DO SUL, 2007). Nesse grupo, foram encontrados 2.550 indivíduos por hectare, pertencentes a 24 espécies e 15 famílias botânicas. As famílias com maior representatividade na área foram Fabaceae e Sapindaceae, sendo que a primeira representou $41,31 \%$ do valor de importância.

Dentre as espécies melhor hierarquizadas destacam-se: Dalbergia frutescens, Aspidosperma australe, Parapiptadenia rigida, Cordia americana e Machaerium stipitatum, as quais perfazem um total de $53,15 \%$ do IVI (Tabela 2). Estas apresentam comportamento pioneiro a secundário (REITZ et al., 1983), o que indica um estágio intermediário de sucessão entre os Agrupamentos 1 e 2. Além disso, o ambiente correspondente a esse agrupamento apresenta menor interferência antrópica que a observada no agrupamento 1 e maior alteração que o agrupamento 2, corroborando o fato de representar uma transição sucessional entre tais ambientes. Entre as espécies, pode-se enfatizar a maior representatividade de Dalbergia frutescens $(\mathrm{IVI}=24,9 \%)$.

A existência dos três agrupamentos foi confirmada a campo pelas suas respectivas estrutura e composições florísticas, caracterizando diferentes estágios sucessionais. Assim, percebe-se, no Agrupamento 1, um estágio menos avançado e com predominância das espécies Ateleia glazioviana e Helietta apiculata, enquanto no estágio sucessional intermediário (Agrupamento 3), essas espécies cedem lugar a Dalbergia frutescens, Aspidosperma australe e Parapiptadenia rigida e, no último e mais avançado estágio (Agrupamento 2), identifica-se a presença de Pilocarpus pennatifolius e Holocalyx balansae, espécies características de estágios sucessionais mais estáveis. Além disso, indivíduos com diâmetros pequenos são característicos de estágios pioneiros a transitórios da sucessão (DAJOZ, 2006). Da mesma forma, menores diâmetros propiciam maior densidade de indivíduos. Assim, o fato de maiores densidades serem encontradas nos Agrupamentos 1 e 3 corrobora a afirmação dos diferentes estágios sucessionais encontrados na área.

O estímulo ao processo de restauração por meio da vegetação, parte da premissa que as plantas constituem a base da cadeia alimentar (TOWNSEND et al., 2006) e dessa forma, sua escolha deve ser direcionada de forma a atingir as condições anteriormente existentes no local (flora, fauna, solo, clima, entre outras). Nesse contexto, a estrutura horizontal da vegetação arbórea pode auxiliar a escolha de espécies para recuperação, considerando o processo de facilitação, que se caracteriza por uma espécie favorecer o aparecimento de outra de estágio mais tardio (CARPANEZZI, 2005). A identificação de agrupamentos permite direcionar melhor a escolha das espécies, considerando sua presença e distribuição em diferentes ambientes da floresta.

A predominância de Ateleia glazioviana no Agrupamento 1, sugere sua utilização como facilitadora da sucessão, associada à Dalbergia frutescens, Helietta apiculata e Parapiptadenia rigida, que, por serem consideradas espécies de estágio inicial (REITZ et al., 1983; RIO GRANDE DO SUL, 2007) e em razão do melhor crescimento, auxiliam na proteção do solo. Ao mesmo tempo, as espécies da família Fabaceae poderão contribuir para aprimorar as condições edáficas, pela fixação de nitrogênio. No entanto, considerando que Ateleia glazioviana é caracterizada como espécie agressiva na vegetação (REITZ et al., 1983), seu uso deve ser estudado e monitorado, buscando entender sua dinâmica no ecossistema e sua eficiência como facilitadora da sucessão, de forma a não inibir o desenvolvimento de outras espécies.

Eugenia uniflora, Prunus myrtifolia e Syagrus romanzoffiana também podem ser indicadas para a recuperação, pois atraem a fauna dispersora, aumentando a chegada de propágulos de diferentes espécies e elevando a diversidade na área. Sebastiania commersoniana e Eugenia uniflora são sugeridas para plantio em áreas com maior influência da umidade, pois apresentam adaptação à saturação hídrica (REITZ et al., 1983). Cupania vernalis e Pilocarpus pennatifolius podem ser utilizadas para o enriquecimento da vegetação em áreas que apresentam estágio mais avançado no processo de sucessão florestal.

Dessa forma, estudos fitossociológicos podem ser utilizados como indicativo para auxiliar nas estratégias de recuperação, permitindo identificar a estrutura da vegetação e servindo como base para a identificação de espécies a serem utilizadas em arranjos, considerando as condições ambientais. No entanto, é necessária a instalação de áreas de pesquisa para fornecer subsídios e informações sobre a aplicação de modelos de recuperação, considerando a atual fragmentação das florestas e a importância das matas ciliares 
como corredores para a manutenção dos processos ecológicos, preservação dos recursos hídricos e de suas funções sociais e ambientais.

\section{CONCLUSÕES}

Os resultados encontrados no estudo permitem concluir que:

Aárea estudadaapresenta três agrupamentos, caracterizados por diferentes espécies e estágios sucessionais, os quais são importantes na indicação de espécies para a recuperação;

Ateleia glazioviana, Helietta apiculata, Dalbergia frutescens e Parapiptadenia rigida são espécies de estágio inicial e apresentam potencial para implantação como facilitadoras da sucessão;

O plantio de frutíferas nativas, como Eugenia uniflora, Prunus myrtifolia e Syagrus romanzoffiana, auxilia a recuperação pela atração da fauna dispersora;

Cupania vernalis e Pilocarpus pennatifolius podem ser utilizadas para o enriquecimento de áreas em estágio mais avançado da sucessão florestal.

\section{REFERÊNCIAS BIBLIOGRÁFICAS}

ARAUJO, M. M. et al. Análise de agrupamento da vegetação de um fragmento de Floresta Estacional Decidual Aluvial, Cachoeira do Sul, RS, Brasil. Ciência Florestal, Santa Maria, v. 14, n. 1, p. 133147. 2004.

ATTANASIO, C. M. Manual Técnico: Restauração e monitoramento da Mata Ciliar e da Reserva Legal para a Certificação Agrícola - Conservação da Biodiversidade na Cafeicultura. Piracicaba: IMAFLORA, 2008. 60 p.

BACKES, P.; IRGANG, B. Árvores do Sul: guia de identificação e interesse ecológico. Santa Cruz do Sul: Instituto Souza Cruz, 2002. 326 p.

BOURGERON, P. S. Spatial aspects of vegetation structure. In: GOLLEY, F. B. (Org.) Tropical rain forest-ecosystems: structure and function. Amsterdam: Elsevier Scientific Publishing, 1983. Cap.3, p. 29-47.

BROWER, J. E.; ZAR, J. H. Field and laboratory methods for general ecology. 2nd ed., Iowa: Brown Publishers, 1984. 226 p.

BUDKE, J. C. et al. Florística e fitossociologia do componente arbóreo de uma floresta ribeirinha, arroio Passo das Tropas, Santa Maria, RS, Brasil. Acta Botanica Brasílica, São Paulo, v. 3, n. 18, p. 581-589, 2004.
CARPANEZZI, A. A. Fundamentos para a reabilitação de ecossistemas florestais. In: GALVÃO, A. P. M.; PORFÍRIO-DA-SILVA, V. (Orgs.) Restauração Florestal: Fundamentos e Estudos de Caso. Colombo: Embrapa Florestas, 2005. Cap.2, p. 27-45.

CARVAlHO, P. E. R. Espécies Arbóreas Brasileiras. Colombo: Embrapa Florestas, 2003. 1039 p. v. 1.

DAJOZ, R. Princípios de Ecologia. Porto Alegre: ARTMED, 2006. 519 p.

DURLO, M. A. et al. A composição e estrutura da mata secundária no vale do rio Jacuí, RS. Ciência e Natura, n. 4, p. 129-139, 1982.

FELFILI, J. M.; VENTUROLI, F. Tópicos em análise de vegetação. Brasília: Universidade de Brasília/Departamento de Engenharia Florestal, 2000. 34 p.

FERRETTI, A. R. Modelos de Plantio para a Restauração. In: GALVÃO, A. P. M.; MEDEIROS, A. C. de S. (Editores Técnicos). Restauração da Mata Atlântica em Áreas de sua Primitiva Ocorrência Natural. Colombo: Embrapa Florestas, 2002. Cap. 4, p. 35-43.

KENT, M.; COKER, P. Vegetation description analyses. London: Behaven Press, 1992. 363 p.

KILKA, R. V. Alguns aspectos florísticos e estruturais de uma floresta de galeria no sul da Planície Costeira do Rio Grande do Sul. 2002. 74 f. Monografia (Bacharelado em Ciências Biológicas)-Universidade Federal de Pelotas, 2002. LEITE, P. F. Contribuicão ao conheciemento Fitoecológico do Sul do Brasil. Ciência \& Ambiente, Santa Maria, v. 1, n. 1, p. 51-73, 2002.

MARIOT, A. A biodiversidade em usinas hidrelétricas. Meio Ambiente. 28 jun. 2007. Online. Disponível em: http://www.revistameioambiente. com.br/2007/06/28/a-biodiversidade-em-usinashidreletricas/. Acesso em: 28 de abril 2009.

MATTEUCCI, S. D.; COLMA, A. Metodologia para el estudio de la vegegetación. Washington: Secretaria General de la Organización de los Estados Americanos - Programa Regional de Desarrollo y Tecnológico, 1982. 169 p.

MORENO, J. A. Clima do Rio Grande do Sul. Porto Alegre: Secretaria da Agricultura, 1961. 42 p. QUADROS, F. L. F. de; PILlAR, V. de P. Transições floresta-campo do Rio Grande do Sul. Ciência \& Ambiente, Santa Maria, v. 1, n. 1, p. 109-118, 2002.

RAMBALDI, D. M.; OLIVEIRA, D. A. S. (Orgs.). Fragmentação de ecossistemas: causas, efeitos 
sobre a biodiversidade e recomendações de políticas públicas. 2. ed. Brasília: MMA/SBF, 2005. 510 p. REITZ, P. et al. Projeto Madeira do Rio Grande do Sul. Itajaí: Herbário Barbosa Rodrigues, 1983. $525 \mathrm{p}$.

RICKLEFS, R. E.; MILLER, G. L. Ecology. 4. ed. New York: W. H. Freeman and Company, 1999 $821 \mathrm{p}$.

RIO GRANDE DO SUL. Governo do Estado, Secretaria Especial do Meio Ambiente. Diretrizes ambientais para restauração de Matas Ciliares. Porto Alegre: SEMA/DEFAP, 2007. 32 p.

ROSA, S. F. et al. Aspectos florísticos e fitossociológicos da Reserva Capão de Tupanciretã, RS, Brasil. Ciência Florestal, Santa Maria, v. 18, n. 1, p. 15-25, 2008.

SANTOS, R. F. (Org.) Vulnerabilidade Ambiental. Brasília: MMA, 2007. 192 p.

SEMA/UFSM-RS. Governo do Estado. Secretaria Estadual do Meio Ambiente. Relatório Final do Inventário Florestal Contínuo do Rio Grande do
Sul. Porto Alegre, Santa Maria: 2002. 706 p. SOBRAL, M. et al. Flora arbórea e arborescente do Rio Grande do Sul. Porto Alegre: RimaNovoAmbiente, 2006. 350 p.

STRECK, E.V. et al. Solos do Rio Grande do Sul. 2. ed. Porto Alegre: EMATER/RS-ASCAR, 2008. $222 \mathrm{p}$.

TOWNSEND, C. R. et al. Fundamentos em Ecologia. Porto Alegre: ARTMED, 2006. 592 p. VACCARO, S. et al. Aspectos da composição florística e categorias sucessionais do estrato arbóreo de três Subseres de uma Floresta Estacional Decidual, no município de Santa Tereza - RS. Ciência Florestal, Santa Maria, v. 9, n. 1, p. 1-18, 1999.

VACCARO, S.; LONGHI, S. J. Análise fitossociológica de algumas áreas remanescentes da Floresta do Alto Uruguai, entre os rios Ijuí e Turvo, no Rio Grande do Sul. Ciência Florestal, Santa Maria, v. 5, n. 1, p. 33-53, 1995.

Ci. Fl., v. 21, n. 2, abr.-jun., 2011 